\title{
A Multiaxial Data-Based Machine Learning Model for Exercise Motion Recognition
}

\author{
Jung-euk Ahn ${ }^{1}$, Eun-Surk $\mathrm{Yi}^{2}$, Ji-Youn $\mathrm{Kim}^{3}$ and Byung Mun Lee ${ }^{4 *}$ \\ ${ }^{1,4}$ Dept. of Computer Engineering, Gachon University, Seongnam-si, Korea \\ ${ }^{2,3}$ Dept. of Exercise Rehabilitation \& Welfare, Gachon University, Incheon, Korea \\ ${ }^{1}$ woodencatty@gmail.com, ${ }^{2} y i e s @ g a c h o n . a c . k r,{ }^{3}$ eve14jiyoun@naver.com, \\ ${ }^{4}$ bmlee@gachon.ac.kr
}

\begin{abstract}
Wearable devices using only accelerometers cannot correctly recognize the detailed motions of exercise performed by patients and cannot count the correct number of repetitions. Therefore, in this paper, we suggest a method to improve the recognition accuracy of detailed motions using machine learning technology simultaneously using an accelerometer, and magnetometer in a wearable device. We particularly improved the recognition accuracy of detailed exercise motions through machine learning using data measured on 9 axes. To verify its effectiveness, we conducted experiments for the recognition of each motion when the patients performed exercise consisting of nine motions with the 9-axis accelerometer attached to their wrists. Accuracy of $81.8 \%$ was recorded when only acceleration data was used, whereas accuracy of $91 \%$ was recorded in the 9-axis data obtained using three sensors, thereby improving accuracy by $9.2 \%$.
\end{abstract}

Keywords: Exercise, Motion Sensor, Recognition, Machine Learning, IoT

\section{Introduction}

Appropriate exercise for hospitalized patients improves prognosis after surgery and helps patients recover rapidly. More specifically, it helps to alleviate symptoms not only of injuries requiring rehabilitation, but also for post-surgery treatment of diseases such as cancer and prevents osteoporosis, fatigue, and some side effects of cardiovascular disease that may occur during treatment[1]. It also helps improve deteriorated physical activity function and relieve psychological depression that may occur due to long-term hospitalization after discharge[2].

However, the patient must exercise according to the prescribed motions and process, and the appropriate amount of exercise depends on the patient's condition and disease severity. If the exercise posture is incorrect or if the exercise motion puts a strain on the surgical site, it may adversely affect recovery[3]. For example, patients who underwent surgery for breast cancer should be careful of chest muscle and shoulder muscle contraction. In other words, customized exercise is necessary for motions such as moving arms or raising the shoulder depending on the prognosis after surgery. If the patient exercises in excessively or with bad posture, it may adversely affect the muscles of the surgical site, which may delay recovery. Therefore, it is necessary for medical staff to prescribe correct exercise motions and process to each patient[4]. However, many medical staff as well as administrative and financial support from medical institutions are needed to prescribe and manage patient exercise for patients who need special rehabilitation, which presents a huge challenge. One way to solve this problem is to use ICT technology, which is used in the field of smart healthcare and life care.

Received (November 5, 2017), Review Result (January 20, 2018), Accepted (January 24, 2018)

${ }^{*}$ Corresponding Author 
With the development of IoT technology in recent years, ICT is evolving into an edge computing technology that handles some of the load of existing cloud computing, which can be realized by machine learning, which is a type of artificial intelligence technology. In other words, complex functions and services that combine miniaturization and intellectualization can be implemented in the edge device. If these technologies can be used to prescribe exercise to patients, customized intelligence services for individual patients will become available. This service can be significantly improved to the level of recognizing and measuring segmented motions or complex motions beyond the level of measuring walking and running activity. It will then become possible to correct patients' erroneous motion or measure the amount of exercise based on measured data, thereby inducing the patient to refrain from excessive exercise.

Many studies have been conducted on human motion, but most of these studies have used only motion sensors equipped with accelerometers. Accelerometers measure based on the state of the device or shock waves recorded by the device. However, their ability to accurately measure patient motion is limited because the device is not able to measure the direction and speed of patients' motion.

Detailed exercise motion may be more accurately measured by using a wearable device that assists in patient exercise and measures their exercise motions along with machine learning. The use of a magnetometer capable of measuring direction and a gyroscope capable of measuring instantaneous acceleration with the wearable device can further improve the accuracy of motion recognition and compensate for the accelerometer's disadvantage of being unable to measure direction and instantaneous acceleration.

To this end, in this study, we designed a wearable device that can be worn by the patient and suggested a model and system that recognize the patient's motion in real time. In particular, we built a learning data set to improve the accuracy of motion recognition and defined a preprocessing technique that processes the data set into a form usable during exercise. The method defined in this paper indexes 9 types of data measured in the wearable device and processes them into one data set. Accuracy can be improved by using this data set with machine learning to learn the motions and using it to recognize the user's motion.

In Chapter 2, we introduce relevant literature, and in Chapter 3 we identify the exercise to be performed by the patient. In Section 4, we present various conditions for data collection by measuring patients' motion using the wearable device. In Section 5, we suggest a method of gathering the collected data and processing it into one data set for machine learning. In Section 6, we design a process to implement machine learning using the data set processed by the suggested method. In Section 7, we discuss the results of having the subjects perform the exercises described in Chapter 3 with wearable devices, measuring the data to implement machine learning, and comparing the performance.

\section{Related Researches}

\subsection{Effects of Exercise}

Cultural anthropologists began to study human movement in earnest around 1950. As a result, they started claiming that human motion is instinct and has the power of natural healing [5]. In the early 2000s when cancer research intensified and treatment technology progressed dramatically, higher survival rates were observed in lung cancer patients who regularly exercised for at least 5 days, at least 30 minutes a day, compared to those who did not exercise. This implies that walking, stretching, and low-intensity muscle exercise not only enhance the basic physical strength of the patient but also accelerate the recovery of the disease, giving various positive effects[6]. 
In some studies, a meta-analysis was conducted on the effects of the exercise program for the purpose of recovery rather than rehabilitation on patients. Some studies have proven that an exercise program not only improves the basic physical strength and resilience of the patient, but also restores muscle strength and damaged muscle in the shoulder. The results of the meta-analysis of patients' changes in physical function after surgery according to the frequency of participation in rehabilitation exercise suggest that the appropriate amount of exercise has a positive effect on the recovery of the patient and thus should be recommended[7].

However, providing such exercise programs to patients is challenging for medical institutions. Despite recognizing the positive effect of exercise programs on recovery, it is difficult for medical institutions to provide physical activities to patients with the absence of exercise programs and tools as well as the spatial constraints[8]. In this situation, patients struggle to obtain information about the exercise, exact method, intensity, and frequency and thus often abandon exercise[9].

In addition, to provide exercise services to patients in hospitals, the hospital should be able to give information about exercise and to arrange manpower to assist with exercise. This means that additional costs will be incurred. Since the patient's exercise must be managed, doctors should be assigned to each patient individually, which is difficult for hospitals to manage in practice[10].

However, through the use of an IoT device equipped with various sensors which can recognize motion, it is possible to prescribe appropriate exercise to patients using smart health care technology and to observe whether they perform exercise as prescribed and guide them to exercise appropriately.

\subsection{Motion Recognition Technology Using Sensors}

In previous motion recognition studies, users' motions were measured by sensor devices or smartphone accelerometer and were recognized based on these measurements [11][12]. Some studies involved exercises such as walking, running, stair climbing, rowing, cycling, and muscle strength exercises in everyday life [13]. In addition, some studies were conducted on inferring users' posture and some experiments conducted to measure standing, sitting, and lying positions to add accuracy to motion recognition [14][15]. Finally, some studies involved complex recognition of user motion and posture using both smartphones and wearable devices equipped with accelerometers [16].

Figure 1 shows which sensor data is used to recognize motions and posture. The unfixed smartphone sensor is mainly used for motion recognition and deduces the vibration pattern from measured acceleration data using the SVM method[17][18][19].

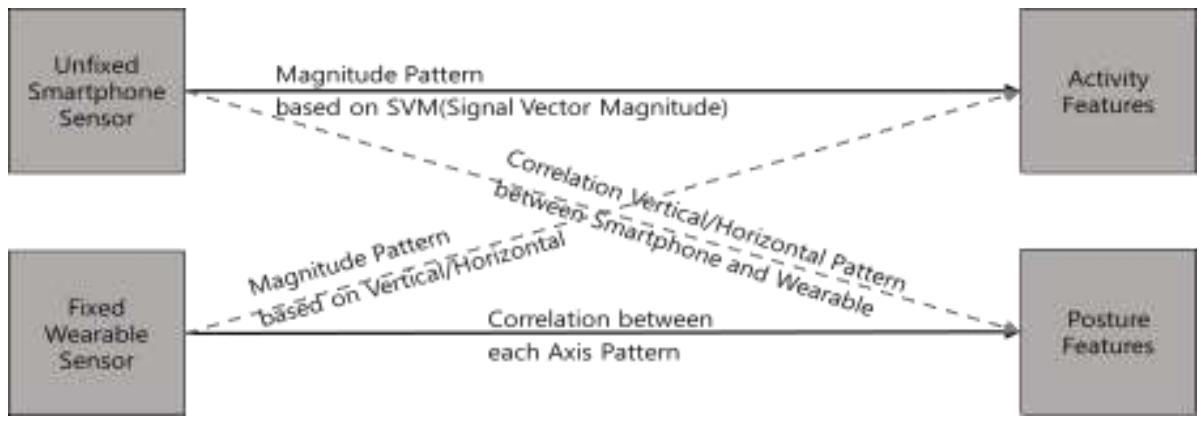

Figure 1. Real-time motion Recognition Structure Using Smart Phone and Wearable Device 
The horizontal/vertical vibration pattern of the fixed wearable sensor is used concurrently to recognize motion. The correction value for each axis of the wearable sensor is used together with vertical/horizontal data patterns for posture recognition.

The process depicted in Figure 1 is needed to recognize 6 types of motion, including walking, stair climbing, sitting, standing, and lying down. We obtained data classified into 10 types as shown in Table 1 below using the accelerometer, gyroscope and magnetometer [20].

For example, body acceleration is the gravitational value of the corresponding region measured using an accelerometer attached to the body. Body angular acceleration is the angular velocity measured using a gyroscope and body angular acceleration magnitude is the angular velocity measured using a geomagnetic sensor.

\section{Table 1. Data Set Items Measured by Sensor}

\begin{tabular}{|l|}
\hline Data name \\
\hline Body acceleration \\
Gravity acceleration \\
Sudden body acceleration \\
Body angular speed \\
Body angular acceleration \\
Body acceleration magnitude \\
Gravity acceleration magnitude \\
Sudden body acceleration magnitude \\
Body angular speed magnitude \\
Body angular acceleration magnitude \\
\hline
\end{tabular}

In these studies, the recognition rate of $88.86 \%$ was increased to $92.49 \%$ compared to when only 3 -axis acceleration was used, but motions could not be effectively recognized because connectivity between motions was not considered in an integrative manner. Pre-processing is required before using measured data from sensors to overcome this difficulty. We can consider using machine learning, i.e. artificial intelligence, for pre-processing [21].

\subsection{Learning Machine}

Machine learning is broadly divided into supervised machine learning and unsupervised machine learning. Supervised machine learning is performed by attaching a label to the learning data set that is the basis for the inference process, and the pattern and distribution form of the data is stored as the results of learning.

These results of learning are later used as the data set. In contrast, unsupervised machine learning is performed without attaching a label to the learning data set. The data is classified according to the degree of similarity between data and the classification criteria are stored as the results of learning and used to identify the data later.

For motion recognition using wearable devices, clear behaviors are targeted with set reasoning, so supervised machine learning is used. It is therefore necessary to measure the motion data by repeating the targeted motions several times with an 
attached wearable device before performing motion recognition and create the learning data set based on the measurement results[22][23].

In all previous studies, supervised machine learning was implemented and various classifiers were used. Classifiers have different characteristics because they use different algorithms for running the learning machine. Therefore, if different algorithms are used for the same data, different recognition accuracy can be recorded. However, all the machine learning algorithms go through the same process and are divided into the training and testing process.

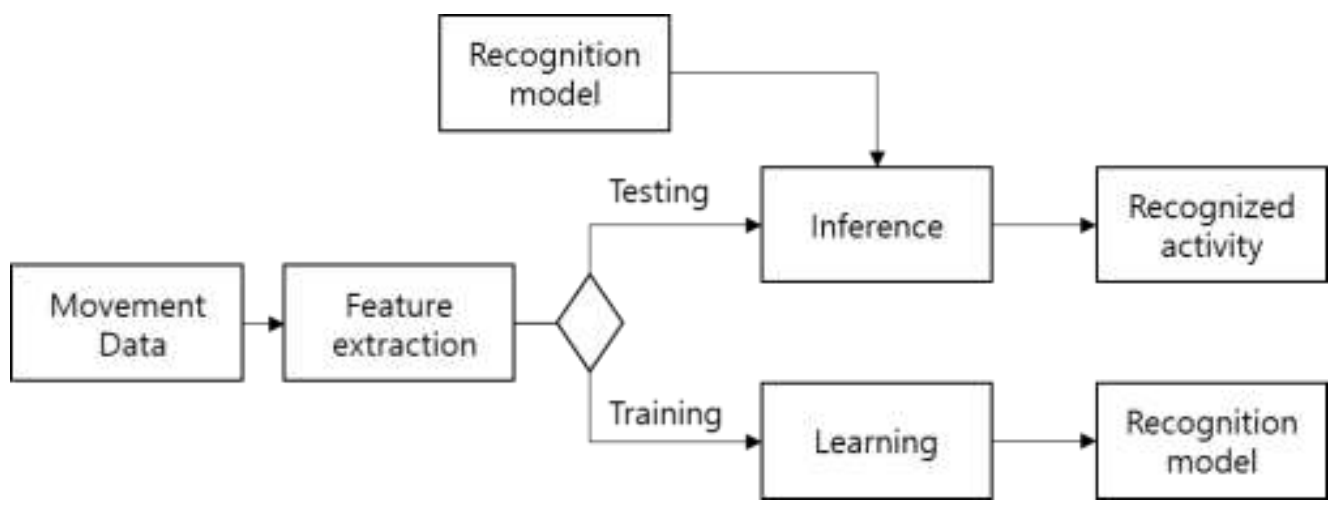

Figure 2. Motion Recognition Processing Using Machine Learning

As shown in Figure 2, we measured data from motions and deduced its characteristics and then built a data set for recognition through the learning process. The motion data measured thereafter can be inferred in the same way. An indicator of performance in this model is whether sufficient data sets are built to prove the reliability of recognition model. More intelligent services can be facilitated if this learning model can be used to evaluate whether patients' exercise motions and load are appropriate.

\section{Smart Recognition Model Using Multiaxial Data}

\subsection{Definition of Exercise Program}

The motion recognition method suggested in this paper is limited to upper body motion to aid in the recovery of breast cancer patients. The reason for this is that the patients who underwent surgery should avoid straining the surgical site when exercising. This allows medical staff to determine the exercise necessary for the patient depending on the type of surgery.

Once the appropriate exercise is determined for the patient, the same approach can be applied to most other patients. As shown in Table 2, exercise was divided into three exercises and seven detailed motions. The three exercises consisted of raising arms forward, clasping hands behind back, and asymmetrically raising arms. The patients repeat each exercise several times at regular intervals depending on their condition. 
Table 2. Exercise Program Model for Breast Cancer Patients

\begin{tabular}{|c|c|c|}
\hline Exercise & Motion & Sequence of motion \\
\hline \multirow{2}{*}{$\begin{array}{l}\text { Raising arms } \\
\text { forward }\end{array}$} & $\begin{array}{l}\text { Raising arm } \\
\text { (front) }\end{array}$ & $\Rightarrow$ \\
\hline & $\begin{array}{l}\text { Lowering arm } \\
\text { (front) }\end{array}$ & \\
\hline \multirow{2}{*}{$\begin{array}{l}\text { Clasping hands } \\
\text { behind back }\end{array}$} & $\begin{array}{l}\text { Clasping hands } \\
\text { behind back }\end{array}$ & \\
\hline & $\begin{array}{l}\text { Clasping hands } \\
\text { behind back and raising }\end{array}$ & $\Rightarrow$ \\
\hline \multirow{3}{*}{$\begin{array}{l}\text { Asymmetrically } \\
\text { raising arms }\end{array}$} & Opening arms & $\Rightarrow$ \\
\hline & $\begin{array}{l}\text { Asymmetrically } \\
\text { raising arms (right) }\end{array}$ & $\Rightarrow$ \\
\hline & $\begin{array}{l}\text { Asymmetrically } \\
\text { raising arms (left) }\end{array}$ & $\checkmark$ \\
\hline Walking & Walking & \\
\hline
\end{tabular}

Looking at the example of raising and lowering arms in Table 2, we can see that patients keep their hands parallel and repeatedly raise and lower them while standing upright. Patients raise their arms until the arms and the body angle are perpendicular. In the clasping hands behind back exercise, the patient puts both hands on the back while standing upright and repeatedly raises both hands to the bottom of the ribs and then lowers them. As this time, the patient should put both hands on the back. When asymmetrically raising arms, the patient spreads their arms horizontally and then raises one arm and lowers the other at the same time, then repeats this motion. At this time, both arms should be kept parallel. Walking was added as an aerobic exercise to help the patient perform lower body exercise and improve their basic physical strength. Since the patient used both arms during the upper body exercises, a wearable device to be attached to the cuff was required to recognize motion and collect the information gathered from it.

\subsection{Learning Machine Model for Exercise Motion Recognition}

In this paper, we suggest a machine learning model using supervised machine learning as shown in Fig. 3, and define it as learning and recognizing motion during exercise. As shown in process (1), the wearable device measures user motion (user action) during exercise. Data on 9 axes is collected from three sensors as shown in Figure 4. The collected data is transmitted to the machine learning application for the use in the machine learning system (2). Process (3) is added to the machine 
learning application for pre-processing prior to data collection. At this time, preprocessing verifies the relation between motions and synchronizes them, playing a pivotal role in improving recognition rate. In process (4), the characteristics are extracted and screened.

Training begins with process (5) in which the data is learned and a recognition model is created, and in process (6), the recognition model is stored for future use. Testing, i.e. process (7), comes after the process (4), and motion is inferred using the recognition model created earlier. In process (8), the motion is finally recognized and the action, which is the results of recognition, is output.

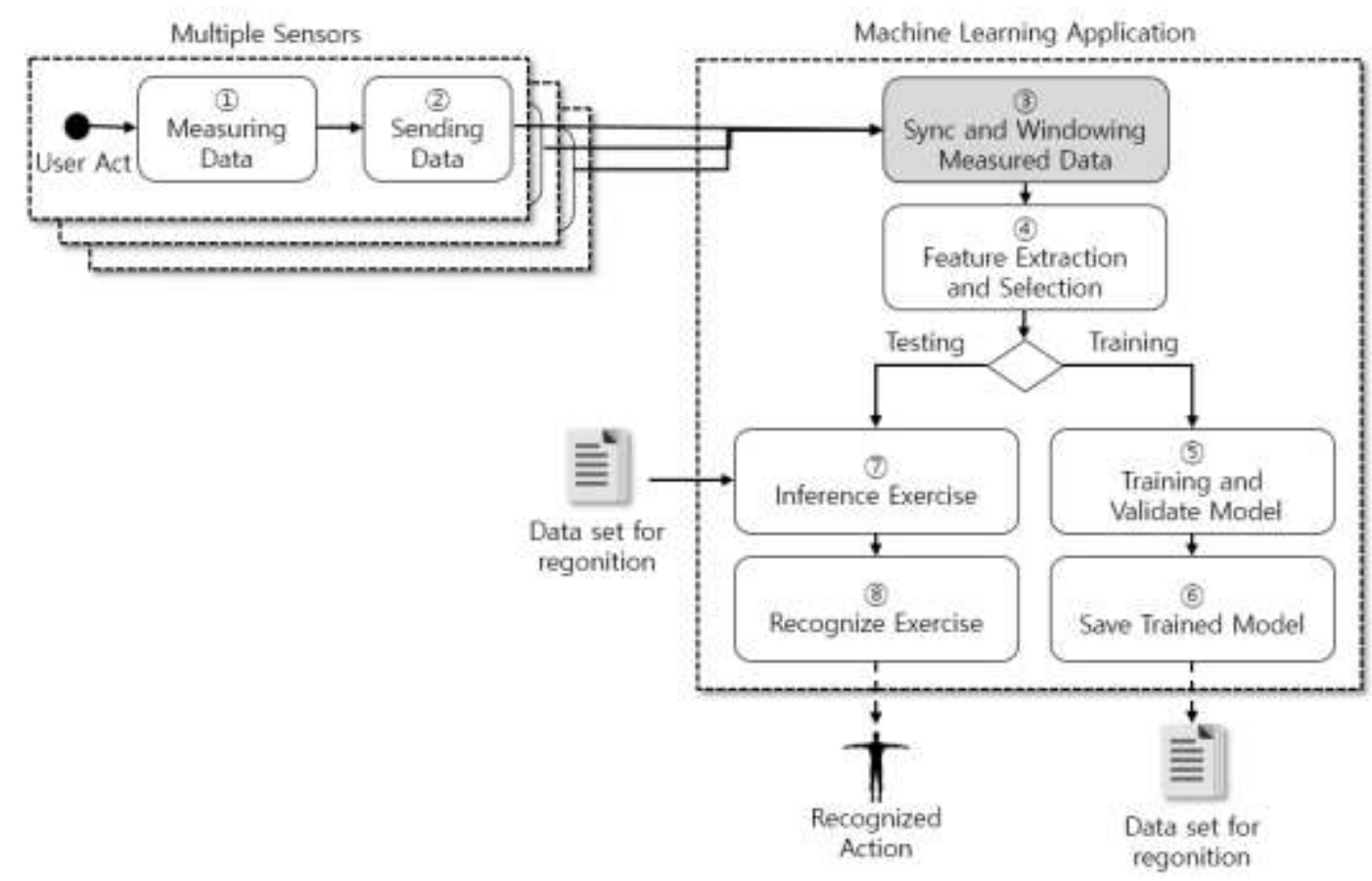

Figure 3. Machine Learning Configuration Model for Motion Recognition

Data measurement and collection

To accurately recognize motion, the direction and speed of the motion should be measured. $\mathrm{x}, \mathrm{y}$, and $\mathrm{z}$ values measured from the accelerometer are used for the speed and the magnetometer is used to measure the direction. The speed of motion can be measured with a gyroscope. Nine types of data can be collected from the three sensors (acceleration $\mathrm{x}, \mathrm{y}, \mathrm{z}$; gyroscope $\mathrm{p}, \mathrm{r}, \mathrm{y}$; geomagnetic $\mathrm{x}, \mathrm{y}, \mathrm{z}$ ) as shown in Figure 4. 


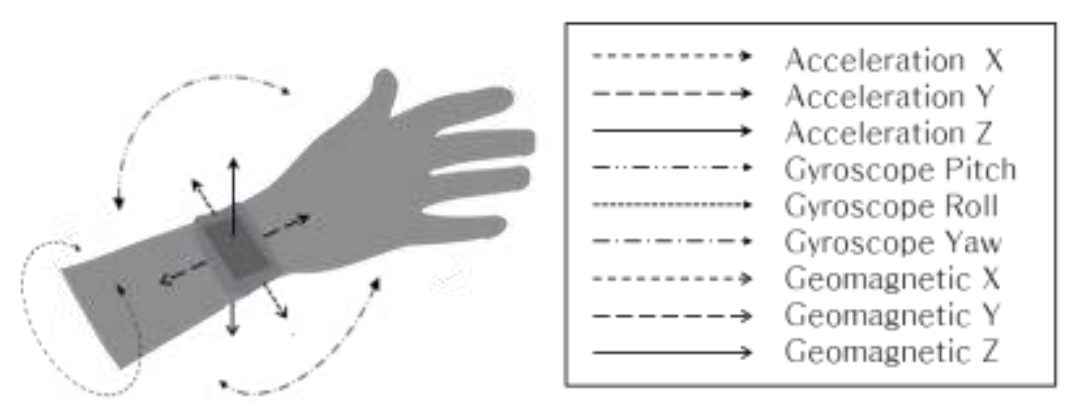

Figure 4. Data Measured using 9-Axis Sensor Device

The data should be measured at regular intervals to maintain accuracy for motion recognition. In this study, the measurement period was set at $100 \mathrm{~ms}$. If the period value is long, the reliability of the data may decrease. In contrast, if the measurement period value is short, the number of data that can be measured per unit time increases, which enables precision measurement and thereby increases accuracy. However, unintentionally measured motion data may make it difficult to deduce characteristics.

In addition, as the gyroscope measures the acceleration of the measured movement, the numerical values of slow limb motion data may be measured inconsistently. When the data measurement period is slower, the resolution of the data deteriorates, so it is difficult to deduce characteristics and to recognize motion accurately during machine learning because the end point of each motion is missed.

\subsection{Data Collection and Synchronization}

To recognize motion using the previously measured data set, all data should be collected and a criterion for dividing the measured data is required. It is necessary to receive and collect the data measured by each wearable device sensor and to attach the index label to each data row so that it can be used for motion recognition. The data set is created by synthesizing collected data. At this time, as shown in Figure 5, nine types of data are grouped together and an index label is attached to manage them. This data set is used for motion data learning or recognition by using a machine learning algorithm later by associating data having the same index label with each other.

The reason for dividing data by index label instead of time unit is to unify data measurement time. The three sensors in the wearable device measure independently, so the measurement period is not perfectly matched. Therefore, when a dataset is created using the time stamp measured by the sensor, there is a problem in that the motion is not recognized as one motion due to a delay time of less than 100 ms even if the data is measured at the same time. To prevent this problem, a delay time of less than $100 \mathrm{~ms}$ is ignored and an index label is attached in the sequence of data measurement to create a data set based on the data measurement sequence. 


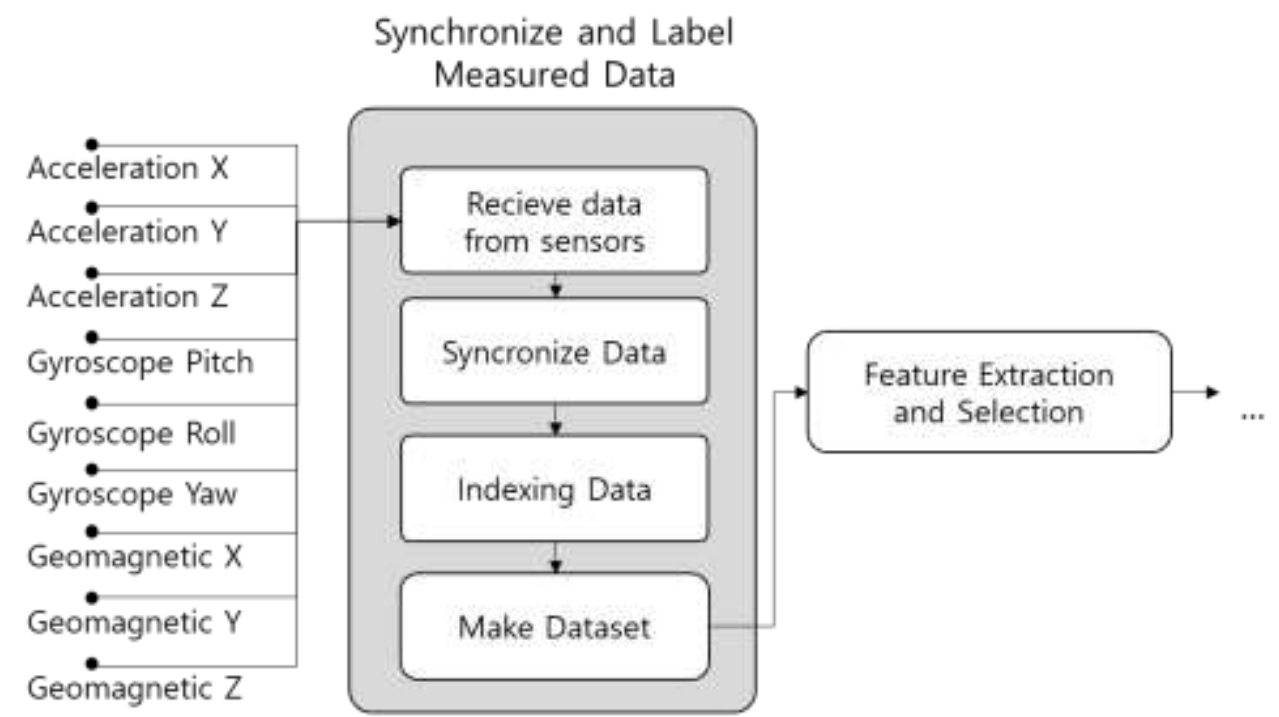

Figure 5. Nine-axis Data Label Integration Process

Acceleration

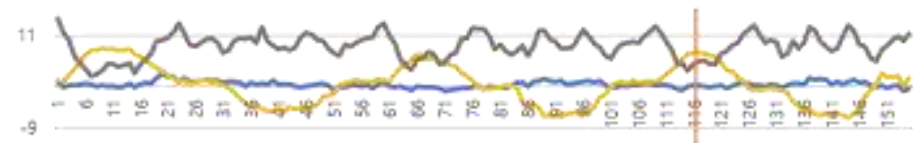

Gyroscope

Geomagnetic

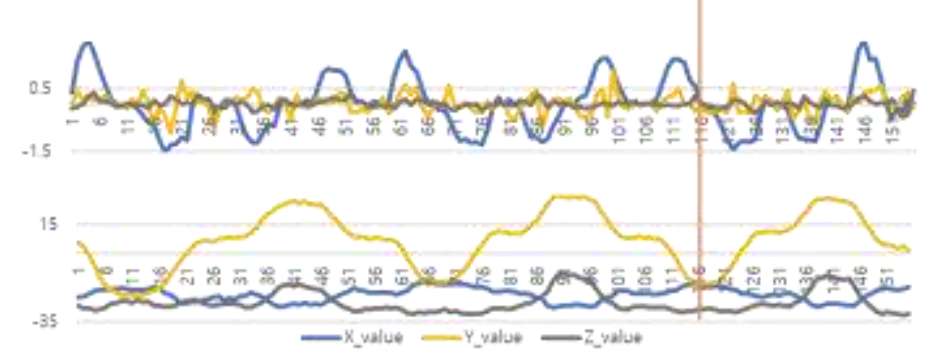

\begin{tabular}{|c|c|c|c|c|c|c|c|c|c|}
\hline Label & \multicolumn{3}{|c|}{ Acceleration } & \multicolumn{3}{c|}{ Gyroscope } & \multicolumn{3}{c|}{ Geomagnetic } \\
\hline \multirow{2}{*}{116} & $\mathrm{X}$ & $\mathrm{Y}$ & $\mathrm{Z}$ & $\mathrm{X}$ & $\mathrm{Y}$ & $\mathrm{Z}$ & $\mathrm{X}$ & $\mathrm{Y}$ & $\mathrm{Z}$ \\
\cline { 2 - 9 } & 0.3382 & 7.23999 & 4.82425 & 0.11026 & -0.11377 & 0.04887 & -16.1175 & -15.8258 & -28.6585 \\
\hline
\end{tabular}

Figure 6. Configuration of Measured Data Set

As shown in Figure 6, a total of 151 pieces of data were collected through measurement. We composed a set using 9 data measured at the 116th among the 151 pieces of data and defined the set as the 116th label. In other words, 116th label identifies all information (motion, direction, speed) about the motion at a specific point of time. The correlation of the measured data appears in the graph in Figure 6. Remembering and learning the changes in each motion can reduce errors in recognizing the next motion. For example, while raising and lowering arms, the patient cannot run at the speed of a 100-meter race, but this may erroneously recognized as 'running fast' in the next motion. In this way, preprocessing with sync and windowing enables more accurate recognition after storing data sets by motion and learning. 


\section{Experiments and Evaluations}

We conducted various experiments to compare the performance of the motion recognition method suggested in the previous section. Machine learning was implemented using the machine learning classifier provided in Weka 3.6.2[24], which is a machine learning tool, and 10-fold cross validation was performed to measure accuracy. The experiment compared the accuracy of the same motion, compared the accuracy when various classifiers were used, and compared accuracy when other people's data was learned.

\subsection{Experimental Environment}

Approximately 4000 rows of learning data were collected and an average of 400 pieces of data per motion were measured to recognize each exercise and its detailed motions. The subjects in this experiment were women in their 50s who were vulnerable to breast cancer. Subjects with wearable devices attached to their wrists performed each motion 20 times. In the case of walking, they walked $100 \mathrm{~m}$ in the usual manner.

The wearable device was replaced by a smartphone and the smartphone was attached to the wrist using bands. LG G6 model smartphones with specifications as in the table below were used. The data was measured using an app called Sensor Kinetics Pro, and data was measured at intervals of $100 \mathrm{~ms}$ after the maximization of sensor performance.

Table 2. Exercise Program Model for Breast Cancer Patients

\begin{tabular}{|c|c|c|c|}
\hline Sort & Name & Vendor & Resolution \\
\hline Device & LG G6 & LG & $*$ \\
Accelerometer & LGE Accelerometer & BOSCH & $0.002 \mathrm{~m} / \mathrm{s}^{2}$ \\
Magnetometer & LGE Magnetometer & AKM & $0.1495 \mu \mathrm{T}$ \\
Gyroscope & LGE Gyroscope & BOSCH & $0.001 \mathrm{rad} / \mathrm{s}$ \\
\hline
\end{tabular}

Exercise motion was measured using the items in Table 3 with standing items added to measure standing posture. Subjects lowered their arms once every 5 times spread to measure arm motion. We did not make any suggestions regarding walking and asked subjects to walk in their usual walking gait. Each motion was performed at intervals of 1 second and was performed more slowly when it was hard to do it.

\subsection{Recognition Accuracy Evaluation by Motion}

Performance was compared between when only acceleration in the same data was used and when all three sensors were used. Figure 7 shows the experimental results evaluated by machine learning using a random tree classifier when only 3 -axis (accelerometer) data was used and when and all 9-axis (accelerometer, gyroscope and magnetometer) data was used. The numerical value is the recognition accuracy of each motion.

The average accuracy was increased by at least $10 \%$ for all motions. The difference was especially pronounced with left-side-up motion. Analysis of the confusion matrix after machine learning implementation showed that the accuracy was decreased because of the confusion between right arm and left arm when only acceleration data was used. This indicates that 9-axis data is more effective than 3-axis data for machine learning. 


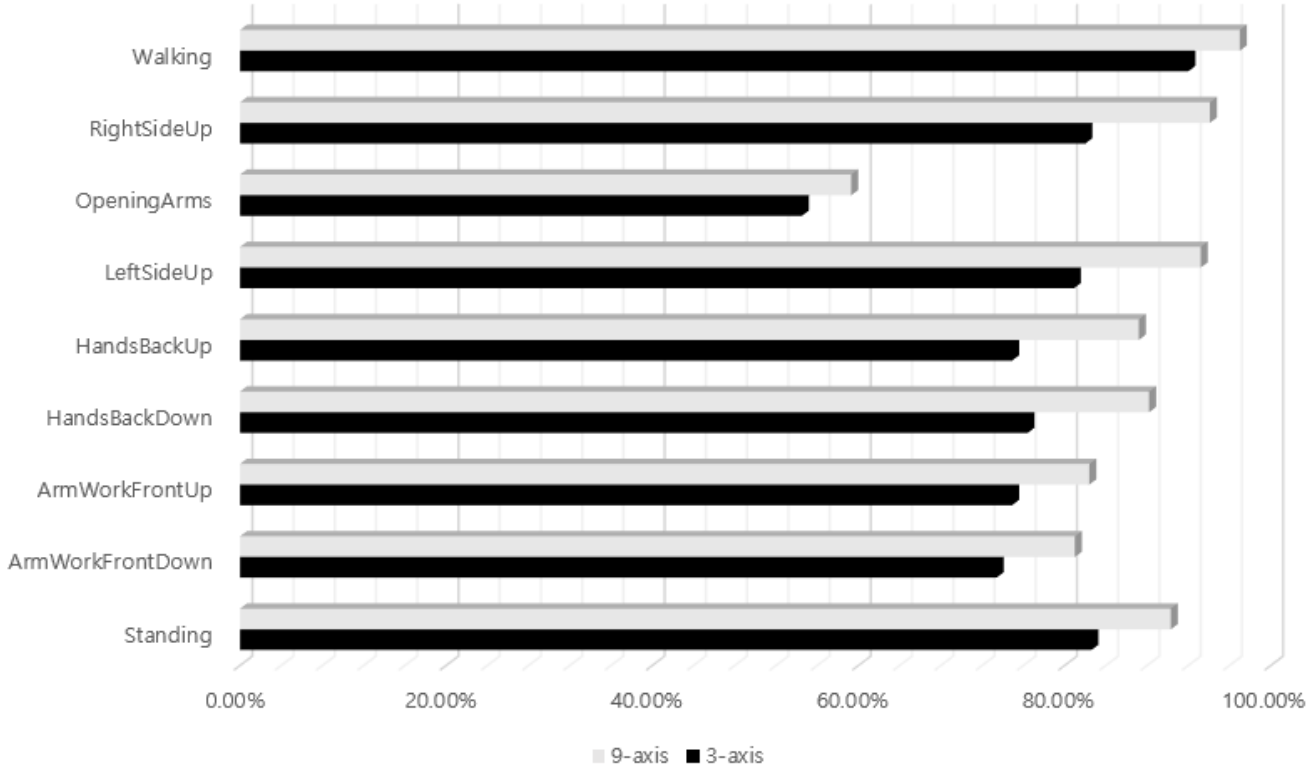

Figure 7. Motion Recognition using Random Tree Classifier

We estimated that this is attributed to the inability to measure the direction or speed of hand motion when only hand motion is measured. In contrast, when magnetometer and gyroscope data are used together with accelerometer data, the whole motion of the arm can be measured. Therefore, the motion can be measured accurately without confusion between the right and the left arm.

\subsection{Accuracy Performance Evaluation by Classifier}

The following is a comparison of performance difference by classifier. Previous data was used and the classifiers frequently used in various studies were selected for comparison and analysis.

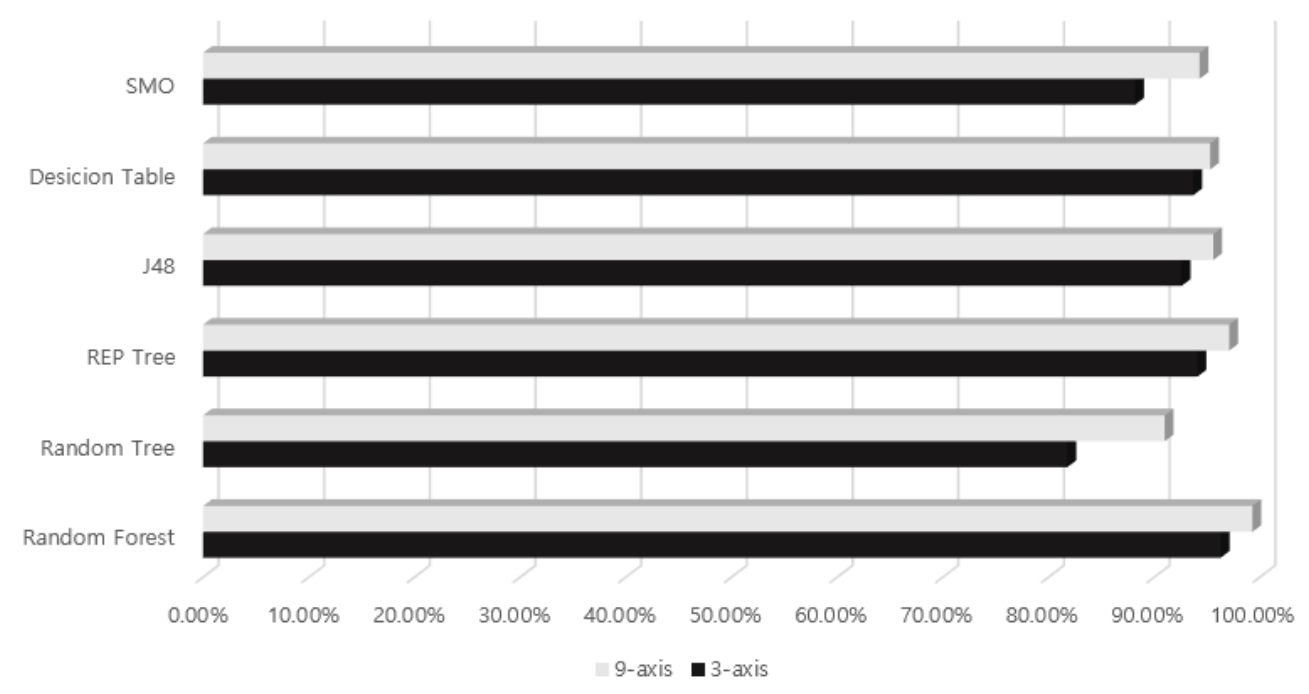

Figure 8. Comparison of Motion Recognition Accuracy by Classifier when the Same Data Was Used 
It required approximately twice as much time to create a recognition model and 10-fold validation for each classifier when a 9-axis sensor was used. This time is required to process more types of data to extract the characteristics of the recognition model. However, when recognition was performed based on the created recognition model, the time required was similar.

\subsection{Versatility Evaluation by Classifier}

Finally, we let the device learn the exercise data of other subjects and recognize motion to compare the versatility of motion recognition. The data from female office workers in their 20s were measured and the same number of motions were measured using the same device. The results showed a more stable wave form.

In most cases, a higher degree of cleanliness was obtained compared to when only the conventional three-axis acceleration was used, but it slightly lagged behind the J48 classifier. However, it was as versatile as the existing ones and there were no overlearning issues that may occur due to excessive data.

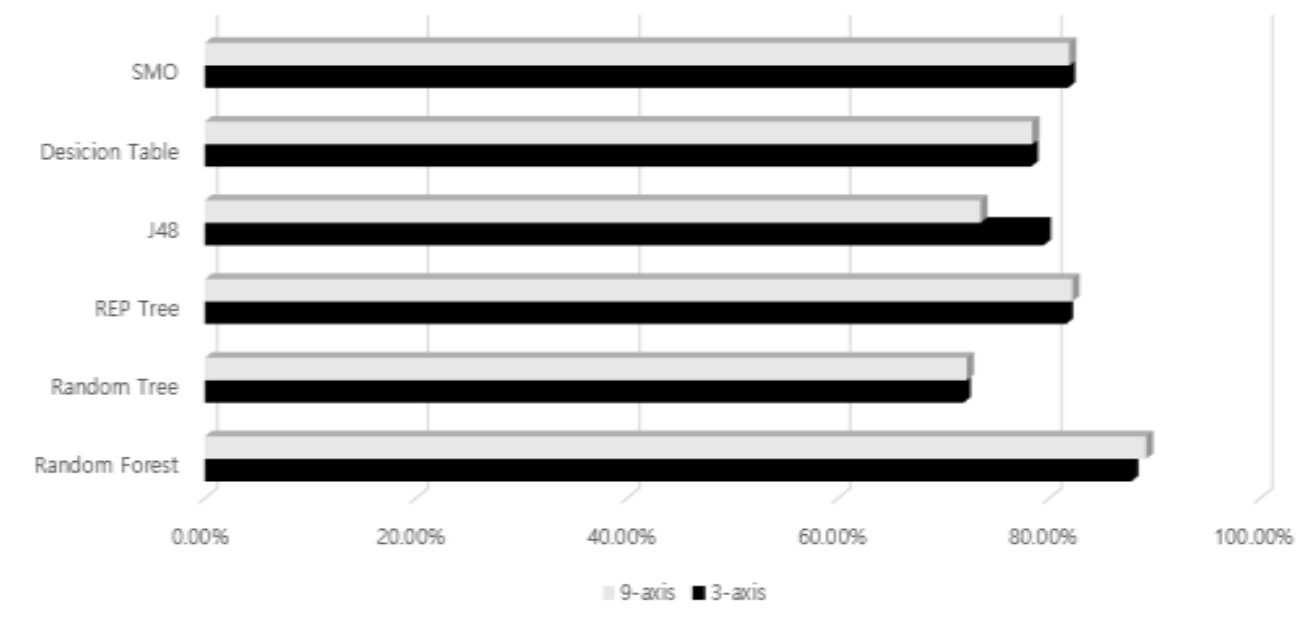

Figure 9. Comparison of Motion Recognition Accuracy by Classifier When Different Data Was Used

\section{Conclusion}

Exercise is an essential element to help patients recover during hospitalization. However, inappropriate exercise can adversely affect recovery, which may lead to severe injuries. To prevent this, it is necessary to measure the motion of the patient during exercise and to provide appropriate feedback. In addition, devices for this purpose should also be versatile enough to recognize exercise motion in response to various physical conditions of the patient.

In this study, we measured the patients' motion during exercise by attaching wearable devices to their wrists and measured their detailed motions to see if the patient exercised with correct posture and performed each exercise the prescribed number of times. The proposed device recognized the exercise motions of patients with different physical conditions by using machine learning, thus ensuring versatility. A gyroscope and magnetometer were used together with the accelerometer to accurately measure patients' motion. The results showed great improvement in recognition accuracy.

Further studies are required of a system that allows patients to autonomously perform an exercise program and receive feedback on the exercise. 


\section{Acknowledgments}

This work was supported by the Ministry of Education of the Republic of Korea and the National Research Foundation of Korea (NRF-2016S1A5B6913737).

This paper is a revised and expanded version of a paper entitled "Customized Exercise Evaluation Model using 9-axis sensors in Wearable Device" presented at 2017 1st International Workshop on Cultural and Technological Exchange and Mutual Development of the Pacific Rim Countries, Cheonan-si, Korea, (2017), December 16-17.

\section{References}

[1] G. C. Kosmadakis, A. Bevington, A. C. Smith, E. L. Clapp, J. L. Viana, N. C. Bishop and J. Feehally, "Physical exercise in patients with severe kidney disease", Nephron Clinical Practice., vol. 115, no. 1, (2010), pp. 7-16.

[2] C. J. Peddle, H. J. Au and K. S. Courneya, "Associations Between Exercise, Quality of Life, and Fatigue in Colorectal Cancer Survivors", Diseases of the Colon \& Rectum., vol. 51, no. 8, (2008), pp. 12421248.

[3] P. E. Peach, "Overwork weakness with evidence of muscle damage in a patient with residual paralysis from polio", Archives of physical medicine and rehabilitation, vol. 71, no. 3, (1990), pp. 248-250.

[4] K. R. Bae, "Development of a posttraumatic growth promoting program for working breast cancer patients", Yonsei University, (2017).

[5] J. Huizinga and F. C. H. Richard, "Homo ludens: A study of the play-element in culture", Routledge \& Kegan Paul., (1949).

[6] M. M. Clark, P. J. Novotny, C.A. Patten, S. M. Rausch, Y. I. Garces, A. Jatoi, J. A. Sloan and P. Yang, "Motivational readiness for physical activity and quality of life in long-term lung cancer survivors", Lung cancer., vol. 61, no. 1, (2008), pp. 117-122.

[7] C. M. Lee, J. H. Lee and D. C. Moon, "The Effect of Exercise Intervention Program in Breast Cancer Patients : A Systematic Review and Meta-analysis", Journal of the Korean society for Wellness, vol. 10, no. 4, (2015), pp. 341-354.

[8] E. J. Yang, S. H. Chung, J. Y. Jeon, K. S. Seo, H. I. Shin, J. H. Hwang and J. Y. Lim, "Current practice and barriers in cancer rehabilitation: perspectives of Korean physiatrists", Cancer research and treatment: official journal of Korean Cancer Association., vol. 47, no. 3, (2015), pp. 370-378.

[9] H. J. Kim, K. K. Park and J. S. Huh, "The Status of Physician Assistants (Physician Assistant) and medical and legal problems", Korean Journal of Medicine and Law, vol. 22, no. 1, (2014), pp. 7-20.

[10] D. H. Kim, "Study on the Supply and Demand for the Physician Manpower in Korea", Health and social science, vol. 8, (2000), pp. 221-239.

[11] M. H. Han and S.Y. Lee, "Personalized Activity Modeling and Real-time Activity Recognition based on Smartphone Multimodal Sensors", Journal of KISS: Software and Applications, vol. 40, no. 6, (2013), pp. 332-341.

[12] E. K. Jeon and H. M. Lee, "Unusual Activity Pattern Detection Application Using Accelermeter Sensor of Moto360", The Korean Institute of Electrical Engineers., Bio-Medical System Conference, (2016), pp. 86-89.

[13] E. M. Tapia, S. S. Intille, W. Haskell, K. Larson, J. Wright, A. King and R. Friedman, "Real-Time Recognition of Physical Activities and Their Intensities Using Wireless Accelerometers and a Heart Rate Monitor, 2007 11th IEEE International Symposium on, (2007), pp. 37-40.

[14] J. Yin, Q. Yang and J. J. Pan, “Sensor-based abnormal human-activity detection”, IEEE Transactions on Knowledge and Data Engineering, vol. 20, no. 8, (2008), pp. 1082-1090.

[15] C. Elkan, "The foundations of cost-sensitive learning", In International joint conference on artificial intelligence, vol. 17, no. 1, (2001), pp. 973-978.

[16] H. S. Lee and S. Y. Lee, "Real-time Activity and Posture Recognition with Combined Acceleration Sensor Data from Smartphone and Wearable Device", Journal of KISS: Software and Applications, (2014), pp. 586-597.

[17] C. M. Jung, J. H. Bang, K. H. Han, H. N. Yum and S. Y. Lee, "Smartphone based activity recognition for real environment", Korean institute of information scientists and engineers Conference, (2013), pp. 460-462.

[18] H. Gjoreski and M. Gams, "Activity/Posture Recognition using Wearable Sensors Placed on Different Body Locations", Proceedings of (738) Signal and Image Processing and Applications, no. 2224, (2011), pp. 716-724.

[19] R. Silva and C. D. Joana, "Smartphone Based Human Activity Prediction", (2013).

[20] D. Anguita, A. Ghio, L. Oneto, X. Parra and J. L. Reyes-Ortiz, "A Public Domain Dataset for Human Activity Recognition Using Smartphones", European Symposium on Artificial Neural Networks proceedings, (2013), pp. 437-442. 
[21] O. D. Lara and M. A. Labrador, "A Survey on Human Activity Recognition using Wearable Sensors", IEEE Communications Surveys and Tutorials, vol. 15, no. 3, (2013), pp. 1192-1209.

[22] U. Maurer, A. Smailagic, D. P. Siewiorek and M. Deisher, "Activity recognition and monitoring using multiple sensors on different body positions", In Wearable and Implantable Body Sensor Networks International Workshop on IEEE, (2006).

[23] O. D. Lara, A. J. Perez, M. A. Labrador and J. D. Posada, "Centinela: A human activity recognition system based on acceleration and vital sign data", Pervasive and mobile computing, vol. 8, no. 5, (2012), pp. 717-729.

[24] S. R. Garner, "WEKA: The Waikato Environment for Knowledge Analysis", In Proceedings of the New Zealand computer science research students conference, (1995), pp. 57-64.

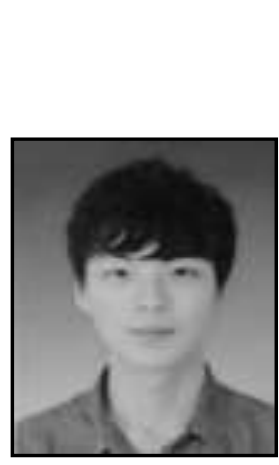

\begin{abstract}
Authors
Ahn Jung-euk, he is currently studing in the department of Computer Engineering, Gachon University, South Korea. His research interests include IoT and Healthcare system.
\end{abstract}

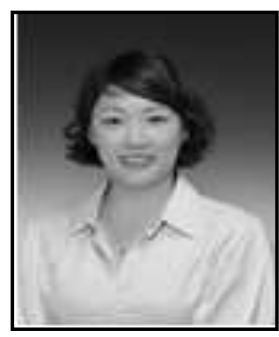

Ji-Youn Kim, she received a B.S. degree in 1998 from Kyusyu woman's College, Fukuoka, Japan and a M.S. degree from Fukuoka Education University and a Ph.D. degree from Sungkyunkwan University, in 2004 and 2008. He is currently a lecturer in the department of exercise Rehabilitation \& Welfare, Gachon University, South Korea. Her research interests are Exercise physiology, Sports nutrition, and health related exercise rehabilitation.

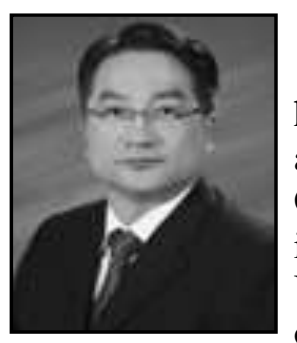

Eun-Surk Yi, he received a B.S., M.S., Ph.D., degree in 1993,1998, 2003 from Korea National Sport, Seoul, Korea. He had worked for professor in the department of Gerokinesiology and Sports Industry, Daegu Haany University for 7 years Gyeongsangbuk-Do, Republic Korea. He is currently a professor in the department of Exercise Rehabilitation \& Welfare, Gachon University, Republic Korea. His research interests are sociology of sports and leisure, activity program protocol for elderly, leisure patterns, convergence Exercise Rehabilitation etc.

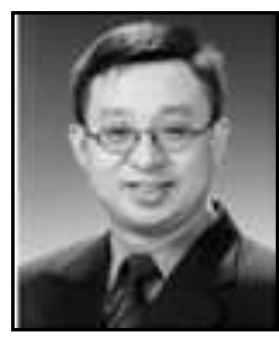

Byung Mun Lee, he received a B.S. degree in 1988 from Dongguk University, Seoul, Korea and a M.S. degree from Sogang University and a Ph.D. degree from University of Incheon Korea, in 1990 and 2007. He had worked for LG Electronics for 7 years. He is currently a professor in the department of Computer Engineering, Gachon University, South Korea. He had been at California State University Sacramento, USA from 2013 to 2014 as a visiting scholar. His research interests are Edge Computing, Wearable IoT Device, IoT for healthcare, wireless sensor networks, operating system, etc. 